\title{
SURVIVAL OF MICRO-ORGANISMS IN SPACE
}

\author{
Results of Gemini-IX-A, Gemini-XII, and Agena-VIII \\ Satellite-Borne Exposure and Collection Experiments
}

\author{
PETER R. LORENZ ${ }^{a}$, JOHN HOTCHIN ${ }^{b}$, ALETHA S. MARKUSEN ${ }^{c}$, \\ GERT B. ORLOBd, CURTIS L. HEMENWAYe, and DOUGLAS S. HALLGREN
}

(Received 12 October, 1967)

\begin{abstract}
Dried suspensions of Penicillitim roqueforti Thom, Coliphage T-1, Bacillus subtilis and tobacco mosaic virus were exposed to space on board the Gemini-IX-A and XII earth satellites and the Agena-VIII space rocket. All micro-organisms tested survived the direct exposure during the GeminiIX-A experiment. In the Gemini-XII experiment only the T-1 phage survived the direct exposure. The survival was influenced by the suspending medium and depended on the species of the microorganism. After four months of space flight on the Agena-VIII space rocket surviving fractions between $2 \times 10^{-3}$ and 1.0 were found in the unopened flight container. However, micro-organisms exposed on the cover of the container during this period were completely inactivated. Shielding against solar ultraviolet radiation during flight resulted in survival of micro-organisms exceeding to that of the transport controls, and the survival was considered complete.

Sterile methylcellulose collection surfaces were exposed to space on board the Gemini-IX-A and $\mathrm{XII}$ satellites in an attempt to collect viable micro-organisms in space. None of the collection surfaces yielded viable micro-organisms.
\end{abstract}

\section{Introduction}

Previous microbiological exposure experiments (НотсніN et al., 1965, 1967a) at altitudes between 34 to $160 \mathrm{~km}$ on board balloons and sounding rockets indicated that short-wave solar ultraviolet radiation which was increasingly absorbed by the atmosphere in the balloon experiments probably accounted for most of the inactivation (LORENZ et al., 1967). These results made it desirable to study the effects of space exposure on micro-organisms flown on board satellites. Satellite experiments allow longer exposure periods than those available in sounding-rocket experiments.

Micro-organisms may possibly remain viable in space for long periods when shielded from lethal radiation by a protective coating (Fedorova, 1963; Hotchin et al., 1967a). Attempts were therefore made to collect and culture possibly viable microbial agents during the Agena-VIII and the Gemini-IX-A and XII space fights.

a Dudley Observatory and Div. of Laboratories and Research of the New York State Dept. of Health.

b Special Projects Research Laboratory, Div. of Laboratories and Research of the New York State

Dept. of Health, and Albany Medical College.

c State University of New York at Albany.

a South Dakota State University. Present address: Dept. of Botany, University of Toronto, Toronto, Canada.

e Dudley Observatory and State University of New York at Albany.

f Dudley Observatory at Albany, New York. 


\section{Materials and Methods}

\section{A. FLIGHT CONTAINERS}

Micrometeorite collectors described elsewhere (HEMENwAY et al., 1967) served as containers for the microbiological experiments. The Gemini 'S-12' collectors (HEMENWAY et al., 1967) consisted of two compartments. This permitted independent closure of one of the compartments accommodating the microbial collection surfaces after ethylene oxide sterilization. Two collectors were prepared for each flight experiment. One served as the 'flight set', another was the 'back-up set' which accompanied the flight set to the launch area and was used as a transport control. Opening and closing of the collectors was done automatically during flight from inside the space capsule.

The Agena micrometeorite collector which was used in the ' $\mathrm{S}-10$ ' experiment (HEMENway et al., 1967) was designed to be opened and closed by hand during the space walk of an astronaut. It was attached to the Agena rocket before launch and consisted of one compartment which accommodated several microbiological exposure and collection experiments in addition to the micrometeorite collection slides. Six microbiological exposure experiments were accommodated on one of the plates fixed to the outside of the cover.

\section{B. MICROBIOLOGICAL PREPARATIONS}

Suspensions of Penicillium roqueforti Thom, Coliphage T-1, Bacillus subtilis and Tobacco mosaic virus (TMV) were seeded on plastic-coated units of the aluminum plates and handled as described below.

Preparation and assay techniques. - Penicillium roqueforti Thom.: Cultures of this mold had been stored at room temperature for 2-7 months to allow sporulation. The spores were eluted with a sterile $1 \%$ Micro-Solv * solution in $\mathrm{H}_{2} \mathrm{O}$.The resulting suspension was centrifuged at $200 \mathrm{~g}$ for $5 \mathrm{~min}$. The pellets were resuspended in physiological saline. The final suspension gave a titer of $3 \times 10^{7} \mathrm{CFU}$ (colony forming units) per $\mathrm{ml}$ and maintained its titer under storage conditions at $4{ }^{\circ} \mathrm{C}$ for approximately 1 year. After flight, the mold was eluted and titrated in physiological saline, and the survivors were determined by colony count after incubation on Sabouraud agar for 2-3 days at room temperature.

Coliphage T-1: The T-1 phage was prepared either in complete broth or in synthetic broth prepared as described below (after BARLow, 1954): Part A: $150.0 \mathrm{~g}$ $\mathrm{Na}_{2} \mathrm{HPO}_{4}$ and $75.0 \mathrm{~g} \mathrm{KH}_{2} \mathrm{PO}_{4}$ dissolved in $1000.0 \mathrm{ml}$ demineralized water. Part $B$ : $41.0 \mathrm{~g} \mathrm{MgSO}_{4} \times 7 \mathrm{H}_{2} \mathrm{O}, 50.0 \mathrm{~g} \mathrm{NaCl}$, and $100.0 \mathrm{~g} \mathrm{NH}_{4} \mathrm{Cl}$ dissolved in $1000.0 \mathrm{ml}$ demineralized water. Dissolve $4.0 \mathrm{~g}$ glucose in a mixture of $40.0 \mathrm{ml}$ of solution part $\mathrm{A}$ and $10 \mathrm{ml}$ of solution part B, dilute in $1950.0 \mathrm{ml}$ of demineralized water and filter sterile. Synthetic broth lysate: Cultures of $E$. coli B were grown in synthetic medium, then inoculated with a $\mathrm{T}-1$ lysate and incubated at $37^{\circ} \mathrm{C}$ on a shaker. The multiplicity of infection was approximately 1 . The resulting lysate was clarified by centri-

* Micro-Solv tissue culture detergent, Microbiological Associates, Inc., Bethesda, Md, 
fugation at $2500 \mathrm{~g}$ for $60 \mathrm{~min}$. and gave a titer of $3 \times 10^{10} \mathrm{PFU}$ (plaque forming units) per $\mathrm{ml}$ on $E$. coli $\mathrm{B}$. Broth concentrate: A T-1 lysate produced from $E$. coli B grown in F-14 A beef extract broth (WHEELER and GrEEN, 1947) was concentrated by centrifugation at $73000 \mathrm{~g}$. The titer showed $10^{11} \mathrm{PFU} / \mathrm{ml}$. Broth lysate: T-1 lysate produced from $E$. coli $\mathrm{B}$ grown in F-14 A beef extract broth (WHEELER and GREEN, 1947) was clarified by centrifugation at $2500 \mathrm{~g}$. Two preparations were used, the titers were $2 \times 10^{9}$ and $3 \times 10^{10} \mathrm{PFU} / \mathrm{ml}$. After the flight experiments, the phage was eluted in F-14 A broth (WHEELER and GREEN, 1947), and the survivors were determined by plaque assay on $E$. coli $\mathrm{B}$.

Bacillus subtilis strain Marburg, ATCC6051: Sterile filtered Pennassay broth (Difco) enriched with garden soil $(1 \%$ ) was inoculated with an overnight Pennassay broth culture and incubated for 48 hours at $32^{\circ} \mathrm{C}\left(\mathrm{H}_{2} \mathrm{O}\right)$ in a shaker bath. The culture was then plunged into an ice bath to prevent further multiplication while serial platings were done on nutrient agar plates to determine the viable cell count. The remaining portion of the culture was then prepared for use in the flight experiments. The nutrient agar plates were incubated for 24 hours at $37^{\circ} \mathrm{C}$. The viable cell count was determined to be $(1.4 \pm 0.03) \times 10^{8} \mathrm{CFU} / \mathrm{ml}$. After flight, the cells were eluted and examined for survivors by colony count.

Tobacco mosaic virus: TMV was grown in Nicotiana tabacum L. var. Havana 38 , extracted from infected leaves, and purified by centrifugation. Pellets of purified TMV were suspended in $0.1 \mathrm{M}$ phosphate buffer at pH 7.0 to a concentration of about $10 \mathrm{mg} / \mathrm{ml}$, and used for seeding the plates. Two sets of controls were kept; one in Albany was used as 'laboratory control' (Table I). The other, in the laboratory in Brookings, S.D., was used as control in the half-leaf assay ('control' Table I). After the experiment, TMV-seeded plates were eluted in test tubes containing $1 \mathrm{ml}$ of $0.1 \mathrm{M}$ phosphate buffer, and the eluate was inoculated to 9-12 half-leaves of $N$. tabacum L. var. Xanthi n.c. Inoculum was applied by capping the forefinger with Saran ${ }^{\mathbf{R}}$ wrap. Handling and inoculation of leaves was done separately by two workers.

Preparation of plates and seeding technique. - An aluminum plate flown in the Agena-VIII collector is shown in Figure 1A. The small squares with circular depressions were plastic coated, autoclaved, seeded using an AGLA* micrometer syringe, and dried at room temperature at a pressure of approximately $0.1 \mathrm{~mm} \mathrm{Hg}$. Suspensions of micro-organisms were seeded either single $(1 \times)$ in $0.02 \mathrm{ml}$ aliquots in the Gemini-IX-A and Agena VIII experiments and in $0.01 \mathrm{ml}$ aliquots in the Gemini-XII experiment, or doubly with $0.01 \mathrm{ml}$ aliquots $(2 \times)$. Half of the aluminum plate was reserved for the collection experiment described below. Altogether 3 plates were seeded. One was used as the laboratory control. It was stored in vacuo $(0.1 \mathrm{~mm} \mathrm{Hg})$ at $4^{\circ} \mathrm{C}$. The other two plates were mounted in the collector box. One was exposed, the other was placed underneath the exposed plate and will hereafter be referred to as 'flight control'.

Figure 1B shows plates used for the Gemini-IX-A exposure and collection experi-

* AGLA micrometer syringe outfit, Burroughs Wellcome \& Company, London, England. 
ments. The circular depressions were acetone cleaned, plastic coated, and autoclaved. Suspensions of micro-organisms were seeded onto the depressions, then dried as described above. Three identical sets of plates were prepared. One was used as flight set, another as back-up set. The third set was used as laboratory control and stored in vacuo at $4^{\circ} \mathrm{C}$. In the flight and back-up sets, plates were mounted in face-up position - 'exposed' and in inverted position underneath the exposed plates - 'flight controls'.

Depressions in sterile, plastic-coated stainless steel plates mounted on the outside

TABLE I

Survival of Tobacco Mosaic Virus ${ }^{\text {a }}$

\begin{tabular}{|c|c|c|c|c|}
\hline \multirow{2}{*}{ Flight Vehicle } & \multicolumn{2}{|c|}{ Number of Lesions } & \multirow{2}{*}{$\begin{array}{c}\text { Average }^{c} \\
\text { Treatment } \\
\text { Control }\end{array}$} & \multirow{2}{*}{$\begin{array}{l}\text { Surviving } \\
\text { Fraction }^{\mathrm{d}}\end{array}$} \\
\hline & Controla $^{\mathrm{a}}$ & Treatment ${ }^{b}$ & & \\
\hline \multirow{12}{*}{ Gemini IX-A } & 43 & 1 & \multirow{3}{*}{0.00002} & \multirow{3}{*}{$0.002^{\mathrm{e}}$} \\
\hline & 28 & 1 & & \\
\hline & 54 & 0 & & \\
\hline & 44 & 12 & \multirow{3}{*}{0.0002} & \multirow{3}{*}{$0.02^{\mathrm{f}}$} \\
\hline & 29 & 4 & & \\
\hline & 61 & 8 & & \\
\hline & 31 & 342 & \multirow{3}{*}{0.01} & \multirow{3}{*}{$1.0^{\mathrm{g}}$} \\
\hline & 18 & 165 & & \\
\hline & 43 & 486 & & \\
\hline & 26 & 0 & \multirow{3}{*}{0.00005} & \multirow{3}{*}{$0.005^{\mathrm{h}}$} \\
\hline & 42 & 3 & & \\
\hline & 59 & 5 & & \\
\hline \multirow{10}{*}{ Gemini XII } & 223 & 0 & \multirow[t]{2}{*}{0} & \multirow[t]{2}{*}{$0^{\mathrm{e}}$} \\
\hline & 187 & 0 & & \\
\hline & 287 & 1905 & \multirow[t]{2}{*}{0.007} & \multirow{2}{*}{$0.09^{f}$} \\
\hline & 181 & 1308 & & \\
\hline & 288 & 1922 & \multirow[t]{2}{*}{0.08} & \multirow[t]{2}{*}{$1.0^{\mathrm{g}}$} \\
\hline & 191 & 1953 & & \\
\hline & 212 & 218 & \multirow[t]{2}{*}{0.01} & \multirow[t]{2}{*}{$0.1^{\mathrm{i}}$} \\
\hline & 207 & 324 & & \\
\hline & 173 & 237 & \multirow[t]{2}{*}{0.02} & \multirow[t]{2}{*}{$0.2^{\mathrm{j}}$} \\
\hline & 260 & 597 & & \\
\hline
\end{tabular}

a Purified virus, stored at $4^{\circ} \mathrm{C}$, diluted to $2 \times 10^{-5}$ in the Gemini-IX-A experiment, to $10^{-5}$ in the Gemini-XII experiment.

${ }^{\mathrm{b}}$ Seeded doubly with $0.02 \mathrm{ml}$ of purified virus in the Gemini-IX-A experiment, seeded singly with $0.01 \mathrm{ml}$ in the Gemini-XI experiment, exposed as indicated.

- Corrected for dilution difference between control and treatment.

d Relative to laboratory controls.

e Directly exposed.

f Flight control

S Laboratory control.

h Back-up set

i Back-up set 'exposed'.

j Back-up set 'flight control'. 
of the Agena-collector cover were seeded with four $0.01 \mathrm{ml}$ layers of suspensions of micro-organisms. Two of the depressions were seeded with mixtures of equal volumes of the T-1 phage broth concentrate and of the Penicillium-spore suspension. The other depressions were seeded with plain suspensions of these micro-organisms. One 'exposed' and one 'laboratory control' plate were prepared for this experiment.
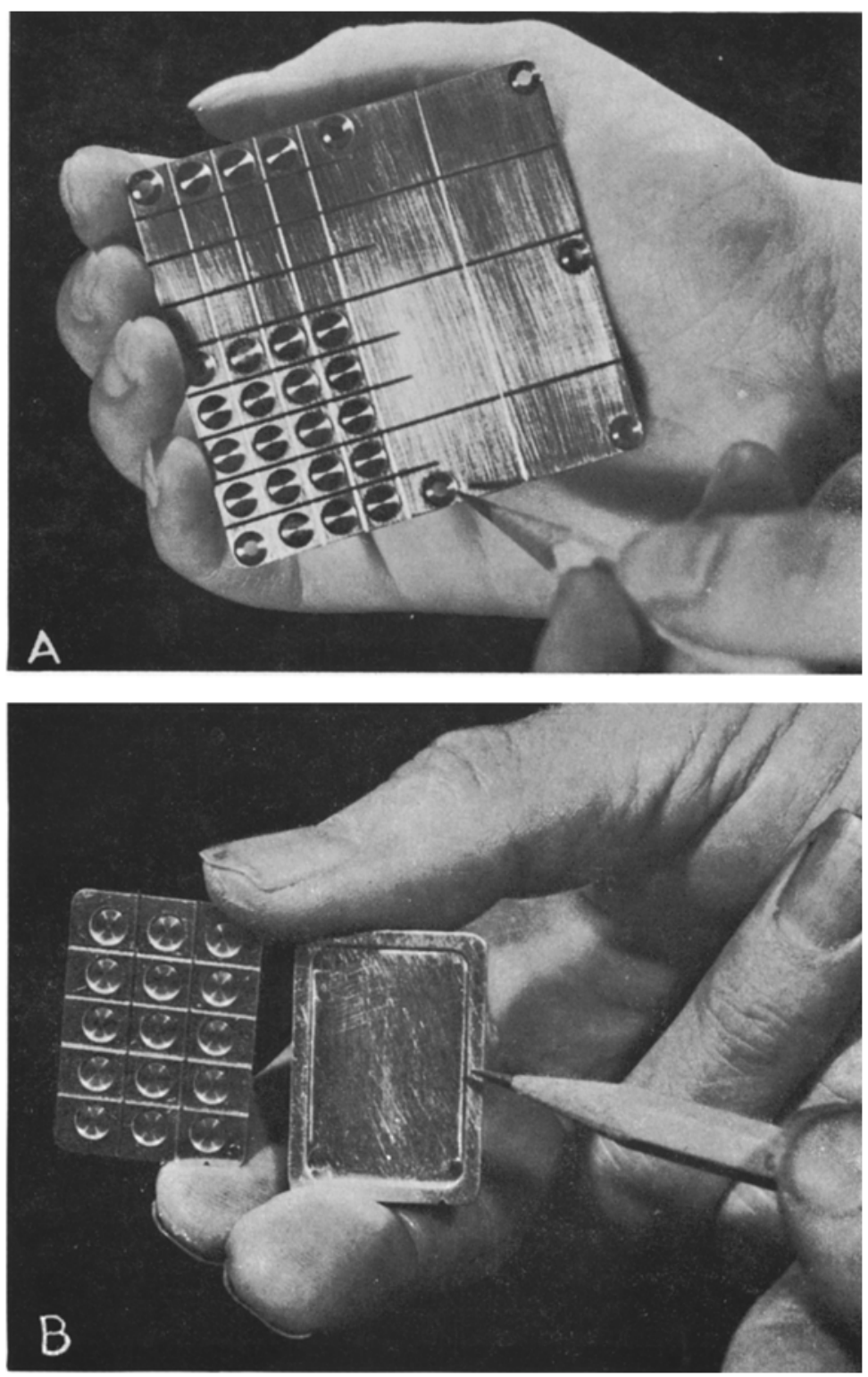

Fig.1. Aluminum plates used for the microbiological exposure and collection experiments in the Agena (A) and Gemini (B) experiments. 


\section{MICROBIOLOGICAL COLLECTION TECHNIQUE}

Two aluminum plates with one large central depression $\left(6 \mathrm{~cm}^{2}\right)$ prepared for the Gemini experiments (Figure 1B) and one half of the Agena-VIII plate (Figure 1A) served as bases for the collection surfaces. Details of the collection techniques are given below.

Preparation of the collection surfaces. - Half of the Agena plate and the large depressions of the Gemini plates were plastic coated, then covered with $1.5 \mathrm{ml}$ of a $7.5 \%$ solution of methylcellulose in $\mathrm{H}_{2} \mathrm{O}$ and immediately frozen with dry ice. The frozen surfaces were lyophilized leaving the methylcellulose as an elastic 'landing' surface. The plates were subsequently autoclaved and immediately vacuum dried at pressures of approximately $1 \mathrm{~mm}$ of $\mathrm{Hg}$.

Sterile handling of the collection surfaces. - The collection surfaces were protected by masks during seeding of the micro-organisms on the Agena plates. The masks were removed inside a dusthood* immediately before the assembly of the flight payload. During the assembly period of 2 hours and during the period of droplet seeding of 2 hours and $45 \mathrm{~min}$, six open blood agar petri dishes served as contamination controls for air-borne micro-organisms. The petri dishes were incubated under aerobic or anaerobic conditions for approximately 1 month. Two colonies were found in the set of petri dishes exposed during seeding; they were identified as Corynebacterium acnes. Three colonies were found in the set of petri dishes exposed during the assembly of the Agena-flight unit. Two of these were identified as coagulase negative Staphylococci, one as Corynebacterium acnes. All these organisms are known as skin contaminants and may have originated from the workers handling the plates.

The collection plates were placed inside the sterile compartment of the Gemini collector, which was then sterilized with ethylene oxide ( 4 hours at $54{ }^{\circ} \mathrm{C}$, approximately 1000 Torr, $40 \%$ relative humidity in an atmosphere of Penn-gas $* *)$. After sterilization the collector was immediately closed by remote control. Then it was transferred into a 'clean' room for the independent loading of the unsterile compartment. Technical details of the sterilization set up and the clean-room facilities are given elsewhere (HEMENwAY et al., 1967).

Elution and assay techniques of the collection surfaces. - The collection surface of the Agena plate was separated under sterile conditions by breaking along the groove separating the exposure and collection parts. The Gemini collectors were introduced into a glovebox, which was equipped with germicidal UV lights and an absolute air filter*** permitting continuous recirculation of the air in the closed system. Simultaneously, autoclaved elution media and tools were introduced into the glovebox. All equipment was exposed to UV irradiation for about $15 \mathrm{~min}$ and to a dust-free filtered air stream for approximately 16 hours in the Gemini-IX-A experiment, and for about 1 hour and $30 \mathrm{~min}$ in the Gemini-XII experiment prior to the opening of the sterile

* Microvoid dusthood, Air Control, Inc., Morristown, Pa., U.S.A.

** Penn-gas, Pennsylvania Engineering Company.

*** Cambridge absolute filter, Cambridge Filter Corp., Syracuse, N.Y., U.S.A. 
compartments. The collection surfaces were then removed from the Gemini collectors and immediately transferred into $5 \mathrm{ml}$ of sterile gelatine tris Hank's (GTA) elution medium, then stored in a refrigerator at $5^{\circ} \mathrm{C}$ until the methylcellulose surfaces had dissolved. Preparations of the resulting suspensions were independently assayed by three groups of researchers for bacterial or fungal growth using the techniques described elsewhere (HotchIN et al., 1967b). Additional studies attempted to isolate animal viruses from the collection surfaces using the techniques described below. The suspensions were inoculated into cultures of primary monkey kidney cells (MK), primary human amnion-tissue cells (HA), a continuous line of human embryonic lungtissue cells (HEL), diploid human lung cells (di HL), and into the amniotic cavities of 10-day old embryonated eggs. The inoculated tissue cultures were observed for cytopathic effects over periods of 10 to 11 days, and blind passages were made to second generations of tissue cultures after incubation in MK, HA and di HL tissue. The second-generation tissue cultures were also observed for 10 to 11 days. The supernatant fluids of the MK-tissue cultures were tested after the incubation periods for hemagglutination of chicken and guinea-pig erythrocytes and the cell sheets were tested for the hemadsorption (HAd) of guinea-pig erythrocytes. The HA cells were tested after the incubation periods for HAd of guinea-pig erythrocytes. Amniotic fluid was harvested after 3 days of incubation of the embryonated eggs, and two more blind passages were made. After each incubation period, the harvested amniotic fluid was tested for hemagglutination of chicken and guinea-pig erythrocytes.

\section{Flight Conditions}

In the Gemini-IX-A experiment the micro-organisms and collection surfaces were exposed to space for 16 hours 47 min under conditions of random tumbling at an average altitude of approximately $300 \mathrm{~km}$. The flight lasted from June $3 \mathrm{rd}$ to $6 \mathrm{th}, 1966$. In the Gemini-XII flight, which started on November 11th, 1966, the biological surfaces were exposed to space for 6 hours $24 \mathrm{~min}$. During this time the samples were faced away from earth at an altitude of approximately $300 \mathrm{~km}$. In the Agena-VIII experiment the micro-organisms on the cover were exposed to the air blast during launching and to space at an average altitude of about $460 \mathrm{~km}$ under conditions of random tumbling for approximately 4 months (March 16th to July 21st, 1966). Due to the emergency change of the flight plan of the Gemini-VIII mission, the collector was not opened and the biological surfaces remained protected by the cover $(7.5 \mathrm{~mm}$ thick aluminum and $2 \mathrm{~mm}$ thick stainless steel). Further details of the exposure conditions are given elsewhere (HEMENWAY et al., 1967).

\section{Results}

No viable micro-organisms were found on the collection surfaces of the Agena-VIII and the Gemini-IX-A and XII experiments, and the detailed results of the tests are therefore omitted. 
TABLE II

Survival of T-1 Phage Broth Concentrate

\begin{tabular}{|c|c|c|}
\hline Flight Vehicle & Surviving $P F U^{a}$ & Surviving Fraction $b$ \\
\hline \multirow{12}{*}{ Gemini VIII Agena } & $0^{c}$ & 0 \\
\hline & $1.3 \times 10^{9 \mathrm{~d}}$ & 1.0 \\
\hline & $3.7 \times 10^{7 \mathrm{e}}$ & 1.0 \\
\hline & $(2.7 \pm 0.8) \times 10^{6 \mathrm{f}}$ & 0.006 \\
\hline & $(4.6 \pm 1.5) \times 10^{5 \mathrm{~g}}$ & 0.09 \\
\hline & $(2.8 \pm 0.6) \times 10^{6 \mathrm{~h}}$ & 0.003 \\
\hline & $(1.8 \pm 1.5) \times 10^{6 i}$ & 0.004 \\
\hline & $(7.4 \pm 2.4) \times 10^{5 j}$ & 0.1 \\
\hline & $(2.5 \pm 1.1) \times 10^{6 \mathrm{k}}$ & 0.002 \\
\hline & $(4.5 \pm 2.0) \times 10^{81}$ & 1.0 \\
\hline & $(5.4 \pm 2.2) \times 10^{6 \mathrm{~m}}$ & 1.0 \\
\hline & $(1.1 \pm 0.1) \times 10^{9 n}$ & 1.0 \\
\hline \multirow{5}{*}{ Gemini IX-A } & $(1.2 \pm 0.8) \times 10^{20}$ & 0.000002 \\
\hline & $(3.8 \pm 2.5) \times 10^{5 p}$ & 0.006 \\
\hline & $(5.8 \pm \mathbf{0 . 7}) \times 10^{7} \mathrm{q}$ & 1.0 \\
\hline & $(1.1 \pm 0.7) \times 10^{5 \mathrm{r}}$ & 0.002 \\
\hline & $(3.6 \pm 1.2) \times 10^{5 \mathrm{~s}}$ & 0.006 \\
\hline \multirow{5}{*}{ Gemini XII } & $(2.8 \pm 2.3) \times 10^{4 t}$ & 0.00003 \\
\hline & $(1.6 \pm 0.1) \times 10^{8} \mathrm{p}$ & 0.2 \\
\hline & $(9.8 \pm 3.6) \times 10^{9 \mathrm{q}}$ & 1.0 \\
\hline & $(5.8 \pm 4.9) \times 10^{7 \mathrm{r}}$ & 0.06 \\
\hline & $(9.6 \pm 0.5) \times 10^{7 \mathrm{~s}}$ & 0.1 \\
\hline
\end{tabular}

a $\mathrm{PFU}=$ plaque-forming units.

b Relative to the laboratory controls.

c Directly exposed on cover of collector, four experiments.

d Laboratory control for plain phage suspension exposed on cover of collector, two experiments.

e Laboratory control for phage suspension mixed with equal volume of Penicillium, two experiments.

f Unopened collector 'exposed', seeded singly with $10^{9} \mathrm{PFU} /$ unit, based on three replicate experiments.

g Like (f), phage 10-fold diluted in F-14 A broth (WHEELER and GreEN, 1947).

n Like (f), seeded doubly.

i 'Flight control' for (f).

j 'Flight control' for $(\mathrm{g})$.

k 'Flight control' for (h).

I Laboratory control for (f).

m. Laboratory control for (g).

n Laboratory control for (h).

- Directly exposed, seeded doubly with $2 \times 10^{9} \mathrm{PFU} / \mathrm{unit}$, based on three replicate experiments.

p Flight control.

a Laboratory control.

r Back-up set 'exposed'.

s Back-up set 'flight control'.

$t$ Directly exposed, seeded singly with $6 \times 10^{\ni} \mathrm{PFU} /$ unit, based on three replicate experiments. 


\section{TABLE III}

Survival of T-1 Phage Synthetic Broth Lysate

\begin{tabular}{|c|c|c|}
\hline Flight Vehicle & Surviving $\mathrm{PFU}^{\mathrm{a}}$ & Surving Fraction ${ }^{b}$ \\
\hline \multirow{6}{*}{ Gemini VIII Agenac $^{c}$} & $(2.9 \pm 1.7) \times 10^{4} \mathrm{~d}$ & 0.007 \\
\hline & $(6.0 \pm 1.4) \times 10^{4 \mathrm{e}}$ & 0.005 \\
\hline & $(6.2 \pm 1.3) \times 10^{4 \mathrm{P}}$ & 0.01 \\
\hline & $(1.4 \pm 0.2) \times 10^{5} \mathrm{~g}$ & 0.01 \\
\hline & $(4.2 \pm 0.9) \times 10^{6 \mathrm{~h}}$ & 1.0 \\
\hline & $(1.1 \pm 0.04) \times 10^{7 i}$ & 1.0 \\
\hline \multirow{5}{*}{ Gemini IX-A } & $0^{\mathrm{j}}$ & 0 \\
\hline & $(4.3 \pm 1.3) \times 10^{2 k}$ & 0.00002 \\
\hline & $(2.2 \pm 0.9) \times 10^{71}$ & 1.0 \\
\hline & $(1.2 \pm 0.9) \times 10^{2 m}$ & 0.000005 \\
\hline & $(7.5 \pm 6.6) \times 10^{1 \mathrm{n}}$ & 0.000003 \\
\hline
\end{tabular}

a See footnotes of Table II.

b See footnotes of Table II.

c Unopened collector.

d 'Exposed', seeded singly with $2 \times 10^{8} \mathrm{PFU} /$ unit, based on three replicate experiments.

e Like (d), seeded doubly.

i 'Flight control' for (d).

$g$ 'Flight control' for (e).

h Laboratory control for (d).

i Laboratory control for (e).

j Directly exposed, seeded doubly with $5 \times 10^{8} \mathrm{PFU} /$ unit, based on three replicate experiments.

k Flight control.

1 Laboratory control.

m Back-up set 'exposed'.

n Back-up set 'flight control'.

\section{TABLE IV}

Survival of T-1 Phage Broth Lysate During the Gemini-XII Flight

\begin{tabular}{lcc}
\hline \multicolumn{1}{c}{ Preparation } & Surviving PFU & Surviving Fraction $^{\mathrm{b}}$ \\
\hline & $9.2 \pm 11.8^{\mathrm{c}}$ & 0.0001 \\
Normal lysate & $(3.1 \pm 2.4) \times 10^{4 \mathrm{~d}}$ & 0.4 \\
& $(7.8 \pm 1.4) \times 10^{4 \mathrm{e}}$ & 1.0 \\
& $(3.0 \pm 0.9) \times 10^{3 \mathrm{f}}$ & 0.04 \\
& $(7.4 \pm 3.8) \times 10^{3 \mathrm{~g}}$ & 0.1 \\
\hline & $(5.1 \pm 5.9) \times 10^{2 \mathrm{~h}}$ & 0.00004 \\
Survivor of direct exposure & $(9.8 \pm 12.9) \times 10^{5 \mathrm{~d}}$ & 0.07 \\
during Gemini IX-A & $(1.4 \pm 0.4) \times 10^{7 \mathrm{e}}$ & 0.07 \\
& $(9.6 \pm 1.1) \times 10^{5 \mathrm{f}}$ & 0.07 \\
\hline
\end{tabular}

a See footnotes of Table II.

b See footnotes of Table II.

c Directly exposed, seeded singly with $2 \times 10^{7} \mathrm{PFU} /$ unit, based on three replicate experiments.

d. Flight control.

e Laboratory control.

I Back-up set 'exposed'.

g Back-up set 'flight control'.

h Directly exposed, seeded singly with $3 \times 10^{8} \mathrm{PFU} /$ unit, based on three replicate experiments. 
TABLE V

Survival of B. Subtilis Marburg in the Unopened Agena-VIII Collector

\begin{tabular}{lcc}
\hline Type of Exposure & Surviving PFU & Surviving Fraction \\
\hline 'Exposed' & $7 \times 10^{1}$ & 0.3 \\
'Flight Control' & $2.3 \times 10^{2}$ & 1.0 \\
Laboratory control & $2 \times 10^{2}$ & 1.0 \\
\hline
\end{tabular}

a Each result is based on three experiments seeded singly with $3 \times 10^{6} \mathrm{CFU} /$ unit.

The survival of the micro-organisms after drying and storage showed variation depending on the seeding technique, the suspension medium and the species of micro-organism. After seeding in two layers, the T-1 phage-broth concentrate showed between $1 \%$ and $100 \%$ survival (laboratory controls Table II). Between $2 \%$ and $5 \%$ of the T-1 synthetic broth lysate survived the drying (Table III). In both preparations, seeding in two half volumes had improved the survival by a factor of approximately 2 . The broth lysate showed survival between $0.4 \%$ and $5 \%$ after drying (Table IV). The tobacco mosaic virus showed similar survival (Table I), and only a fraction of approximately $10^{-4}$ of the $B$. subtilis had survived (Table V). The Penicillium showed survival between $5 \%$ (Gemini IX-A) and 100\% (Gemini XII).

The exposure results are summarized in Tables I-VI. Data based on three or more replicate experiments were compared using a $t$-significance test. All results are expressed in terms of average numbers of surviving micro-organisms \pm standard deviations and of surviving fractions relative to the survival of the laboratory controls.

T-1 phage broth concentrate: This phage suspension as well as a 1:1 mixture of it with the Penicillium-spore suspension were directly exposed on the Agena-collector cover. Suspensions of this phage were flown in the Gemini and inside the unopened Agena collectors. No survival was found of the phage fully exposed to launch conditions and to space for 4 months on the cover of the Agena collector (Table II). In the Gemini-IX-A and XII experiments, survival rates of $2 \times 10^{-6}$ and $3 \times 10^{-5}$ respectively were found after direct exposure to space. The phage flown on the flight-control plate was approximately 200 -fold inactivated in the Gemini-IX-A experiment, and 5-fold in the Gemini-XII experiment. The back-up sets showed surviving fractions of 0.006 ('flight control') and 0.002 ('exposed') for the Gemini-IX-A experiment, and 0.06 and 0.1 for the Gemini-XII experiment. Between $0.2 \%$ and $10 \%$ of the phage flown in the unopened Agena collector survived.

T-1 phage synthetic broth lysate: This phage suspension was seeded on plates flown in the Gemini-IX-A and Agena collectors. No survival was found after the direct exposure in the Gemini collector (Table III). The survival of the back-up set varied between $5 \times 10^{-6}$ ('exposed') and $3 \times 10^{-6}$ ('flight control'). The survival inside the unopened Agena collector varied between $7 \%$ and $0.6 \%$.

T-1 phage broth lysate: Two broth lysate preparations were flown on board Gemini XII. One was a 'normal' T-1 lysate, the other a broth lysate prepared from a survivor of the direct exposure of the Gemini-IX-A experiment chosen at random. The survival after direct exposure was $1 \times 10^{-4}$ for the normal lysate and $4 \times 10^{-5}$ for 
TABLE VI

Survival of Penicillium Roqueforti Thom.

\begin{tabular}{|c|c|c|}
\hline Flight Vehicle & Surviving $\mathrm{CFU}^{\mathrm{a}}$ & Surviving Fraction ${ }^{b}$ \\
\hline \multirow{6}{*}{ Gemini VIII Agena } & $0^{c}$ & 0 \\
\hline & $9.1 \times 10^{4 d}$ & 1.0 \\
\hline & $5.3 \times 10^{4 \mathrm{e}}$ & 1.0 \\
\hline & $(1.5 \pm 0.1) \times 10^{5 f}$ & 1.0 \\
\hline & $(1.5 \pm 0.4) \times 10^{5 \mathrm{~g}}$ & 1.0 \\
\hline & $(1.2 \pm 0.05) \times 10^{5 \mathrm{~h}}$ & 1.0 \\
\hline \multirow{5}{*}{ Gemini IX-A } & $1.7^{\mathrm{i}}$ & 0.00003 \\
\hline & $(3.6 \pm 0.6) \times 10^{4 j}$ & 0.7 \\
\hline & $(5.4 \pm 2.0) \times 10^{1 \mathrm{k}}$ & 1.0 \\
\hline & $(2.0 \pm 0.7) \times 10^{31}$ & 0.04 \\
\hline & $(1.2 \pm 0.8) \times 10^{3 \mathrm{~m}}$ & 0.02 \\
\hline \multirow{5}{*}{ Gemini XII } & $0^{\mathrm{n}}$ & 0 \\
\hline & $2.1 \times 10^{\tilde{n} j}$ & 0.4 \\
\hline & $5.5 \times 10^{5 \mathrm{k}}$ & 1.0 \\
\hline & $1.9 \times 10^{51}$ & 0.3 \\
\hline & $1.1 \times 10^{5 \mathrm{~m}}$ & 0.2 \\
\hline
\end{tabular}

a $\mathrm{CFU}=$ colony-forming units.

b Relative to the laboratory controls.

c Directly exposed on cover, four experiments.

d Laboratory control for plain Penicillium suspensions, exposed on cover of collector, two experiments.

e Laboratory control for Penicillium suspension mixed with equal volume of T-1 phage broth concentrate, two experiments.

i Unopened collector 'exposed', seeded singly with $10^{6} \mathrm{CFU} /$ unit, based on three replicate experiments.

g 'Flight control' for (f).

h Laboratory control for (f).

i Directly exposed, seeded doubly with $10^{6} \mathrm{CFU} /$ unit, based on the survival of $1 \mathrm{CFU}$ in three replicate experiments.

j Flight control.

k Laboratory control.

1 Back-up set 'exposed'.

m Back-up set 'flight control'.

n Directly exposed, seeded singly with $3 \times 10^{5} \mathrm{CFU} /$ unit, based on two replicate experiments.

the Gemini-IX-A survivor (Table IV). Due to the relatively low precision of the data, this difference is not significant. The survival of the flight and back-up set controls was between 10 to 25 -fold less than that of the laboratory controls.

Penicillium roqueforti Thom.: Dried films of this mold and a $1: 1$ mixture of it with T-1 phage broth concentrate were exposed on the cover of the Agena collector. The suspension was also flown inside the unopened Agena collector and in the Gemini collectors. After 4 months of space exposure no micro-organisms were found alive (Table VI). In the Gemini-IX-A collector, one CFU had survived the direct exposure to space. No survival was found after the Gemini-XII exposure. Between the survival of the Gemini-IX-A and Agena-VIII flight and laboratory controls no significant differences were found. The back-up set showed approximately 25 -fold inactivation 
in comparison to the laboratory controls, there was no significant difference between the 'exposed' and 'flight-control' plates. The survival of the Gemini-XII flight and backup set controls did not vary significantly, it was approximately 3 -fold less than the laboratory controls. No loss of viability was found after 4 months of space flight inside the unopened Agena collector.

Tobacco mosaic virus: TMV was exposed in the Gemini collectors; the results are shown in Table I. After the direct exposure on board Gemini IX-A a fraction of 0.002 had survived. Survival on the flight-control plate was 10-fold higher. The back-up set showed a surviving fraction of 0.005 . No survival was found after the Gemini-XII exposure. The survival of the flight and back-up set controls was 5 to 15-fold less than that of the laboratory controls.

Bacillus subtilis Marburg: A suspension of $B$. subtilis was flown in the unopened Agena collector. During the space flight of 4 months the titer of the 'exposed' plate was reduced to 0.3 (Table V). No titer loss was found on the 'flight-control' plate.

\section{Discussion}

These results confirm and extend the previous conclusion based on brief rocket flights, that terrestrial micro-organisms may survive in space to a much greater extent than we had expected (Hotchin et al., 1967a). They are also of value for spacecraft sterilization and planetary contamination. It is interesting to note that, with the exception of the T-1 phage broth concentrate, nearly all the backup set controls of the GeminiIX-A experiment showed less survival than the flight controls. In the Gemini-XII experiment, on the other hand, the survival of these controls was in most cases comparable. This indicates that in the Gemini-IX-A experiment the transport conditions were more harmful to the micro-organisms than during the Gemini-XII experiment.

It is clear that only very small fractions of the micro-organisms in the Gemini collectors had survived the direct exposure to space. The survival was influenced by the composition of the suspending media. Complete inactivation was found of the T-1 synthetic broth lysate, while the T-1 phage broth suspensions survived to some extent. The chance of survival was obviously increased by screening ingredients in the broth medium. The observation that a survivor of the $\mathrm{T}-1$ phage broth concentrate directly exposed to space in the Gemini-IX-A experiment did not show higher survival in the Gemini-XII experiment than the 'normal' $T$ - 1 phage preparation indicates that the survival of the Gemini-IX-A exposed phage was not based on a few microorganisms of this species specifically resistant to the space radiation.

Some species are apparently more resistant than others. Tobacco mosaic virus was the most resistant micro-organism in the Gemini-IX-A experiment. However, it did not show any survival in the Gemini-XII experiment. During the Agena experiment, $P$. roqueforti, which is known to be very resistant to both drying and storage (HoTCHIN et al., 1967a), was not at all affected and $B$. subtilis only slightly.

The exposure conditions of the micro-organisms flown outside on the Agena- 
collector cover for 4 months represent a considerably longer exposure period than the relatively short exposures in the Gemini experiments. However, it must be kept in mind that these preparations were also exposed to the heat and air blast phenomena during launching of the rocket. It is therefore at the present time not possible to identify the specific cause of inactivation. Considering the collection experiments, the small probability of survival after the direct exposure and the small area of the collection surfaces could explain these results.

The lethal factor which caused the high inactivation of the directly exposed microorganisms could effectively be screened out during the long-term exposure by the cover of the Agena collector. This shield completely screened out solar UV radiation which probably was the main cause of inactivation, as observed in earlier experiments (LORENZ et al., 1967). On the other hand, the high survival of the cover protected micro-organisms in the Agena experiment and the survival of the flight controls in the Gemini experiments suggests that cosmic rays and X-rays resulting from cosmic-ray interaction with the cover or the plates were not responsible for the lethal effect.

\section{Acknowledgements}

This work was supported by the National Aeronautics and Space Administration with grant NsG 155-61 and contract NAS 9-5637.

The authors acknowledge the significant contributions of astronauts R. Schweickart, T. Stafford, E. Cernan, J. Young, M. Collins, J. Lovell and E. Aldrin; further the technical assistance of Mr. J. Jacobs, Miss D. Darcangelis, Mr. M. Colucciello, Mrs. J. Gardner, Mr. K. Stinner, Miss M. Cordato and Mr. B. Burrows; the contributions of Mr. R. Coon, Mr. L. Bourdillon and Mr. T. Laudate to the design of the flight containers and the assembly of the flight payloads, and the co-operation of Mr. J. B. Van Derwerken in the sterilization of the flight containers.

\section{References}

Barlow, J. L.: 1954, Dissertation, School of Hygiene and Public Health, Johns Hopkins University. Fedorova, R. T.: 1963, in Life Sciences and Space Research, vol. II. North-Holland Publishing Company, Amsterdam, p. 305.

Hemenway, C. L., Hallgren, D. S., Coon, R. E., and Bourdillon, L. A.: 1967, Technical description of the Gemini S-10 and S-12 micro-meteorite experiments, COSPAR Symposium, London, England.

Hotchin, J., Lorenz, P., and Hemenway, C.: 1965, Nature 206, 442.

Hotchin, J., Lorenz, P., Markusen, A., and Hemenway, C.: 1967a, in Life Sciences and Space Research, vol. V. North-Holland Publishing Company, Amsterdam, p. 1.

Hotchin, J., Lorenz, P., Markusen A., and Covert, S.: 1967b, The microbiological flora of the Gemini IX space craft before and after flight. NASA Technical Note (in print).

Lorenz, P. R., Hemenway, C. L., and Hotchin, J. : 1967, The biological effectiveness of solar electromagnetic radiation in space, COSPAR Symposium, London, England.

WheEler, M. W. and Green, E.: 1947, in Standard Methods of the Division of Laboratories and Research of the New York State Department of Health (ed. by A. B. Wadsworth). The Williams and Wilkins Company, Baltimore, 3rd ed., pp. 168-237. 